\title{
EVOLUTION
}

INTERNATIONAL JOURNAL OF ORGANIC EVOLUTION

PUBLISHED BY

THE SOCIETY FOR THE STUDY OF EVOLUTION

\begin{tabular}{lll}
\hline Vol. 39 & September, 1985 & No. 5 \\
\hline
\end{tabular}

Evolution, 39(5), 1985, pp. 949-964

\section{HIERARCHICAL SELECTION THEORY AND SEX RATIOS. II. ON APPLYING THE THEORY, AND A TEST WITH FIG WASPS}

\author{
Steven A. Frank \\ Division of Biological Sciences, University of Michigan, Ann Arbor, MI 48109-1048
}

\begin{abstract}
Predictions from the theory of sex ratios in subdivided populations are tested by studying fig wasps (Agaonidae). Observations strongly support the qualitative prediction that fig wasp sex ratios (males/total) decrease with increasing amounts of both inbreeding and competition among male relatives for access to mates (local mate competition). However, the observed sex ratio is consistently lower than predicted by previous quantitative models. Many assumptions underlying these models are unrealistic. Each unrealistic assumption is discussed as it applies to fig wasps, and where appropriate, new quantitative predictions are derived based on more realistic assumptions. New predictions are compared to the data in an a posteriori fashion and are found to be much closer to the observations than previous models from the literature, but further work will be required before a close match between theory and observation can be claimed.
\end{abstract}

Received September 6, 1984. Accepted May 31, 1985

Sex ratio has become a touchstone in the study of adaptation. The theory of sex ratios is rich with predictions, since the proportion of resources allocated to male and female reproductive functions often has a very strong and easily recognized association with reproductive success (Charnov, 1982). Because of this theoretically strong association, the match between theory and observation is an indicator both of the quality of our reasoning in the study of adaptations and of our ability to apply this reasoning successfully to real-life situations. Empirically, the literature provides a large source of measured sex ratios, since sex ratios are both strikingly obvious and relatively easily measured traits, and they are often of great practical significance. On the whole, broad comparative evidence seems generally supportive of predicted qualitative trends (Charnov, 1982). The next round of work on sex ratios must be detailed studies of particular organisms aimed at a priori tests of quantitative predictions. A few notable efforts have recently been made in this direction, and the results are again encouraging, but prompt further work. For subdivided populations, which are the subject of this paper, Hamilton (1979) has reported a small data set for fig wasps, and Werren $(1980,1983)$ has presented a series of elegant lab experiments on the parasitic wasp Nasonia vitripennis that provides a good match with quantitative sex-ratio predictions.

Sex-ratio theory for subdivided populations has been exceptionally prolific and controversial. A general approach that unifies sex-ratio theory of subdivided populations is presented in a related 
paper (Frank, 1985b; see also Nunney, 1985): in such populations three processes cause sex-ratio biases. First, when there is competition among relatives of the same sex for a limiting resource, the sex ratio tends to be biased towards the sex with the lower average relatedness among competitors; that is, the reproductive (or genetic) valuation is lower for the sex with more competition among relatives (Hamilton, 1967; Clark, 1978; Bulmer and Taylor, 1980). Second, inbreeding biases the sex ratio towards females when there is no paternal investment in offspring except sperm, since a daughter that inbreeds passes on one set of parental genes plus a second set with a probability proportional to the level of inbreeding, while a son passes on only one set of parental genes (Fisher, 1941; Charlesworth and Charlesworth, 1978, 1981; Taylor, 1981). Third, if the parent controlling the sex ratio is asymmetrically related to sons and daughters, then the reproductive valuations of sons and daughters are weighted by the regression coefficients of relatedness of the controlling parent to sons and daughters, respectively (Hamilton, 1972, 1979; Trivers and Hare, 1976; Uyenoyama and Bengtsson, 1981, 1982). It is also shown in a related paper (Frank, 1985 b) that group selection (Hamilton, 1979; Colwell, 1981; Wilson and Colwell, 1981) and the genetic correlations described in the first two processes listed above are alternative descriptions of the same phenomenon.

I set out to test specific predictions of this theory by studying fig wasps. In each generation a small number of alreadymated females enter, oviposit, and then die within the fig. Their progeny emerge several weeks later and mate among themselves within the sealed cavity of the fig. The wingless males then chew an exit tunnel through the wall of the fig and die soon after. The winged, already-mated females fly off carrying pollen from the fig, find a new receptive fig, and begin the cycle again.

Fig wasps are haplodiploid: unfertil- ized eggs give rise to haploid males, and fertilized eggs develop into diploid females. With this genetic system, a mother may adjust the sex ratio of her brood by fertilizing or not fertilizing eggs with sperm she stores in a sac (spermatheca) (evidence reviewed in Charnov, 1982). This sex-ratio control makes haplodiploid wasps ideal for testing predictions, since sex ratios can potentially be adjusted to local conditions in a phenotypically plastic manner.

Because of the wasps' natural history and genetic system (further details below), there is some inbreeding in each generation; probably the only form of between- or within-sex competition is competition among males for mates (local mate competition-LMC); and a mother is more closely related to a daughter than to a son.

This study was designed to test a quantitative theorem of sex ratios in which there is local competition among males for mates, inbreeding, and an asymmetry in the relatedness of parents to sons versus parents to daughters (Hamilton, 1979). This sex-ratio theorem is

$$
r^{*}=(1 / 2) R P_{d t}
$$

where $r^{*}$ is the predicted sex ratio, $R$ is Hamilton's (1979) coefficient of inheritance asymmetry between the sexes, and $P_{d t}$ is Wright's (1969) index of panmixia for the locus controlling the sex ratio (see Frank, 1985b). $R=2 B_{p s}\left(B_{p s}+B_{p d}\right)$, where $B_{p s}$ and $B_{p d}$ are the "complete" coefficients of relatedness of parent to son and parent to daughter, respectively (Hamilton, 1972). For haplodiploid organisms with maternal control of the sex ratio, $R=(1+F) /(1+2 F)$ (Hamilton, $1972)$, where $F$ is Wright's (1969) fixation index, a function of the amount of inbreeding (inbredness, see Frank, $1985 b$ ), and $0 \leq F \leq 1$ so that $2 / 3 \leq R \leq$ 1. For diploid organisms there is no asymmetry of inheritance, no matter what the value of $F$, so $R=1$. Formally, $P_{d t}$ is the expected within-deme (within-fig) variance in additive genotypic values divided by the population variance, and 
$0 \leq P_{d t} \leq 1$. By construction of the model, $P_{d t}$ measures both the intensity of local mate competition and the level of inbreeding, while $R$ measures the asymmetry of inheritance between the sexes, so these factors, which affect the sex ratio, are clearly separated (Frank, 1985b; the alternative interpretation of $P_{d t}$ as a group selection term is discussed at length in this related paper-throughout the present paper I will mainly use genetic correlations). For fig wasps, both $F$ and $P_{d t}$ depend on the number of already mated females (foundresses), $n$, that colonize each fig per generation. Because of the fig wasps' unique natural history, the foundresses oviposit simultaneously within a tiny central cavity of the fig, so each foundress is likely to be able to assess the number of foundresses, $n$; and because of the haplodiploid genetic system, each foundress may potentially adjust her sex ratio according to $n$.

When $n$ is small, sib mating is frequent, so $F$ is large, and $R$ is small. $P_{d t}$ is also small, since the amount of sib mating and sib competition is high, making the within-deme variance relatively low. Thus, when $n$ is small, the sex ratio is predicted to be female biased. As $n$ increases, $F$ approaches zero and $P_{d t}$ approaches one, so the predicted sex ratio steadily increases toward $1 / 2$. With certain simplifying assumptions commonly employed in the literature (such as random settling of foundresses onto figs, and others to be discussed at length),

$$
F=1 /(4 n-3), \quad P_{d t}=(n-1) / n,
$$

and

$$
r^{*}=(1 / 2)[(4 n-2) /(4 n-1)][(n-1) / n]
$$

as reported by Hamilton (1979), Taylor and Bulmer (1980), and Uyenoyama and Bengtsson (1982). Uyenoyama and Bengtsson (1982) showed, with different techniques and under slightly different model assumptions, that (2) is actually unstable to all other sex ratios. Biological interpretations of this result remain unclear: for example, a complex evolution- ary dynamic may ensue, or the result may be stable on a small neighborhood surrounding (2).

The data I have collected on fig wasps match very well the qualitative trend that the sex ratio increases as $n$ increases, but the sex ratio is consistently more femalebiased than predicted by Equation (2) (Fig. 1). Several of the assumptions underlying (2) are unrealistic and have a significant effect on the predicted sex ratio, although the general trend is unaffected.

The purpose of this paper is to present some extensions of the quantitative model that are more realistic, make several a posteriori comparisons of the revised theory with the fig wasp data, and discuss further extensions of the theory and possible tests with fig wasps. The a posteriori models are much closer to the observations than previous models from the literature, but further work will be required before a close match between theory and observation can be claimed. Fig wasps are particularly appealing for these studies because their unique natural history allows designed experiments to be conducted on wild populations, and because there are approximately 900 species of fig wasps, an array that will provide a rich source of comparative information.

\section{Fig Wasp Natural History}

The fig wasp Pegoscapus assuetus Grandi (see Wiebes, 1983, for taxonomic commentary) pollinates the fig tree Ficus citrifolia P. Miller. This fig species is a member of the monoecious New World section Urostigma, so the natural history description is of this group (see Frank, $1983 a, 1984)$. It is generally accepted that each fig tree species (Ficus spp.) has its own unique species of pollinator wasp (Agaonidae) (Wiebes, 1979).

A monoecious fig is a globular inflorescence containing hundreds of staminate and fewer pistillate florets lining a nearly sealed cavity. A small number of females enter the fig through a narrow passage (ostiole), and once inside, never leave. The wasps are about $1.5 \mathrm{~mm}$ in 
length, and the fig is 4-6 $\mathrm{mm}$ in diameter at this stage. The females pollinate many of the pistillate florets with pollen carried from the fig from which they emerged, lay an egg in the single-seeded ovary of some of the pistillate florets, and then die. Each larva develops by eating the tissues associated with the developing seed or the seed itself. About one month later the next generation of wingless males emerges and mates with eclosed females while the females are still in the individual florets. A male gains access to a female within a floret by chewing a hole through the wall of the ovary and inserting his telescoping abdomen. Usually 1-4 females enter each fig $(97 \%$ of figs in Ficus citrifolia, Frank, $1983 a$ ), and one-quarter or more of the progeny within a fig are likely to be sibs. After mating the males chew an exit tunnel through the wall of the fig. The mated females leave their natal florets, collect pollen from the now mature staminate florets, then leave through the exit tunnel to find a receptive fig and begin the cycle again. Further details on fig wasp natural history can be found in Hill (1967), Galil (1977), Wiebes (1979), Janzen (1979), and Frank (1984).

\section{Sex-Ratio Experiments}

Sex-ratio data were obtained from $\mathrm{Pe}$ goscapus assuetus, the pollinator of Ficus citrifolia, at experimentally manipulated foundress numbers. These studies were conducted in the Everglades National Park from May to July 1982.

Sex Ratio Versus Foundress Number. - Twigs bearing 10-50 figs too young to have been entered by pollinating wasps were bagged with a fine mesh cloth (Tergal). When the figs became receptive to entering pollinator wasps, the bag was removed and 1-8 pollinator wasps were introduced into each fig. The pollinator wasps were obtained for introductions by rearing wasps from maturing figs on trees within approximately a $10-\mathrm{km}$ radius of the site of the experimental trees. The introductions were accomplished by placing a wasp on a receptive fig and observing whether the wasp entered the fig, walked off, or flew off. If the wasp entered the fig, an entry for that particular fig was recorded; if she walked off the fig, she was replaced on the fig repeatedly for about 5 minutes, and then if she still had not entered, she was discarded; and if she flew off, a new wasp was placed on the fig. This procedure was repeated until the desired number of wasps had entered a particular fig. All introductions into a single fig were performed during a 90 minute period. Each wasp that entered a particular fig was reared from a different maturing fig, guaranteeing that no two foundresses within an experimental fig were sibs. (An experiment will be described below in which only wasps reared from the same maturing fig were introduced into each receptive fig.) After pollinator wasps were introduced into the receptive figs, the Tergal bag was replaced over the twig to exclude additional pollinator wasps and all parasitic wasps from the figs. Parasites commonly oviposit in a fig, greatly reducing the number of pollinator wasps reared. The bag was kept in place for 15-20 days after introductions and then removed.

About 27-32 days after the introductions, the experimental figs began to mature. These figs were collected before the exit tunnel was chewed by the pollinator males, guaranteeing that all male and female pollinator wasps of the progeny generation were collected with each fig. The wasps were reared, and the sex ratios counted. The sex ratio rises steadily with foundress number (Fig. 1), in qualitative agreement with the prediction from Equation (2).

Sex Ratio with Sibling Foundresses. The number of foundresses per fig, $n$, is positively associated with the relative genetic heterogeneity in the deme, $P_{d t}$. (The variance of a small sample is less than the population variance, or, in this case, the most important factor is that the proportion of interactions among sibs decreases as $n$ increases.) However, other cues, such as odors, may provide a better estimate than $n$ of the relative genetic heterogeneity (i.e., kin recognition; see 


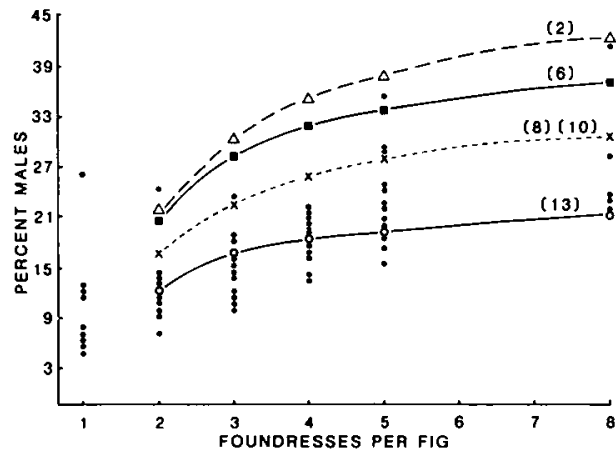

FIG. 1. Predicted sex ratios as a function of the number of foundresses per fig. The upper (dashed) curve is from Equation (2), the middle (solid) curve from Equation (6), and the middle (dotted) curve from Equation (8). This last curve is also almost indistinguishable from Equation (10). The lower (solid) curve is fitted from Equation (13). The assumptions underlying each predicted curve are explained in the text.

Holmes and Sherman, 1982; Blaustein, 1983; Lacy and Sherman, 1983).

To test whether sex ratios are conditional on cues of genetic heterogeneity other than foundress number, a modified experiment was performed. In the previous experiment, each foundress introduced into a particular fig was reared from a different maturing fig, thus guaranteeing that no two foundresses were sibshere these figs that have five foundresses are designated "control figs." In the present experiment, 15 foundresses all reared from the same maturing fig were introduced into three different receptive figs, with five foundresses per receptive fighere these figs are designated "experimental figs." For the experimental figs, there are three replicates from each maturing fig, and the entire procedure was repeated for 12 maturing figs (summary of design in Fig. 2). The proportion of foundresses that are sibs in the experimental figs depends on the unknown number of mothers for their natal fig, but the expected genetic variance within these replicates is certainly lower than in the control figs with five foundresses reared from different maturing figs.

If sex ratios are conditional on genetic

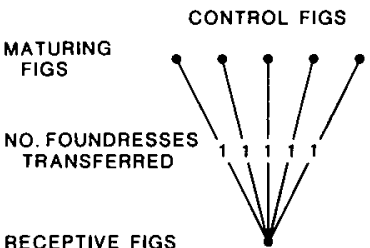

EXPERIMENTAL. FIGS

RECEPTIVE FIGS
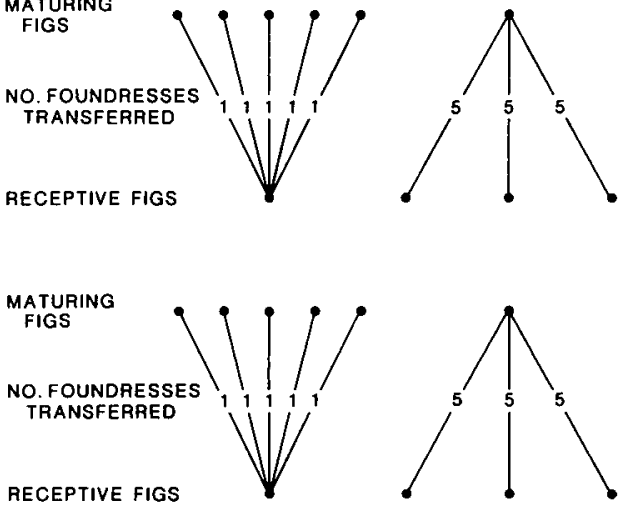

REPEAT DESIGN

:

:

FIG. 2. Schematic summary of two sex-ratio experiments. "Control figs" is an experiment in which five foundresses were introduced into a single receptive fig, and only one of the five foundresses was reared and transferred from each maturing fig. This guarantees that no two foundresses within a receptive fig are sibs. "Experimental figs" is an experiment in which three sets of five foundresses, all reared from the same maturing fig, were reared and transferred into three receptive figs. The three receptive figs used for each maturing fig are referred to as "replicates" of that maturing fig. For the experimental figs, the expected number of sibs per receptive fig is $5 / m$, where $m$ is the number of foundresses per maturing fig. $m$ varies among maturing figs, and in this particular experiment $m$ is not known.

recognition, three a priori hypotheses follow from the theory. i) The median sex ratio per fig will be lower in experimental figs than in control figs. ii) The distribution of sex ratios will have a stronger left-skew in experimental figs than in control figs, since the sex ratio (proportion of males) is predicted to decrease as the proportion of sibs increases within a fig with five foundresses, and the proportion of sibs will vary. iii) The average variance in the sex ratio in experimental figs among the three replicates taken from each maturing fig (within-replicate variance) will be less than the variance in mean sex ratio among maturing figs (among maturing fig variance). This is predicted since some maturing figs would have had few foundresses giving rise to 


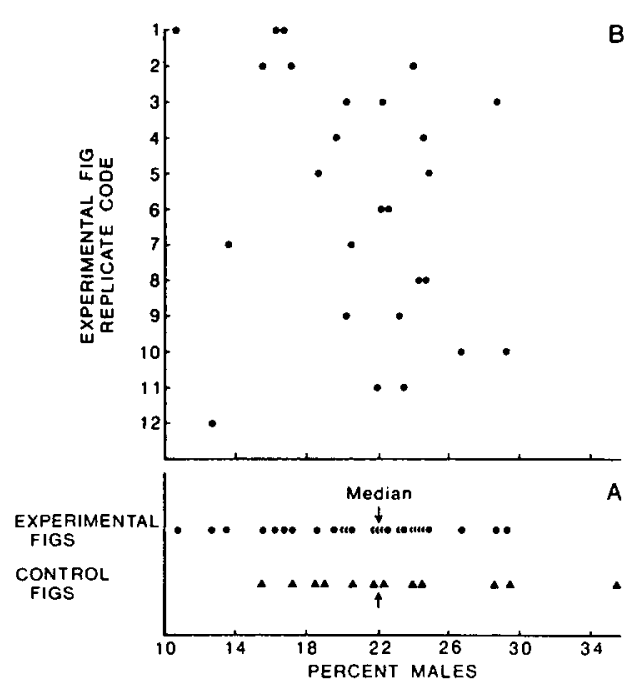

FIG. 3. Sex ratios from figs with five foundresses. A) Experimental figs versus control figs. The medians for the two sets are identical, and the experimental figs have a nonsignificant left skew relative to the control figs $(P=0.43$, KolmogorovSmirnov two-sample test, see text). B) Experimental fig sex ratios displayed by sets of replicates from each of the twelve maturing figs used. The variance among replicates within each maturing fig is less than the variance over all experimental figs, but the difference is nonsignificant according to a KruskalWallis test $(P=0.14$, see text). Missing data were caused by failure of some figs to rear any wasps. See Figure 2 for further details of the design.

replicates with a high proportion of sibs, while other maturing figs would have had many foundresses and, thus, replicates with a low proportion of sibs (refer to summary of design in Fig. 2).

Analyses of the three hypotheses are discussed in turn. i) The median of the sample of control figs is 0.221 (sample of 12) and the median of the sample of experimental figs is also 0.221 (sample of 26) (Fig. 3A). Clearly, no shift in the median sex ratio has occurred. ii) The only standard procedure available for testing a shift in skew between two populations when the control is not a priori symmetric is the Kolmogorov-Smirnov two-sample test (procedure outlined in Hollander and Wolfe, 1973). The alternative hypothesis is one-sided since the prediction is that the experimental population will exhibit a greater left-skew (towards low sex ratios, i.e., few males) than the control population. The test statistic is $J_{2}^{\prime}=0.533, P=0.43$ (data in Fig. $3 \mathrm{~A})$, so there is not sufficient evidence to reject the null hypothesis. However, the Kolmogorov-Smirnov test is notoriously weak (i.e., high type II error rate), and if a real difference exists between the control and experimental populations, it is likely to be small. iii) The hypothesis that in experimental figs the average variance among replicates from the same maturing fig is less than the variance over all experimental figs can be studied by a Kruskal-Wallis test (Hollander and Wolfe, 1973). The test statistic is $H=$ 16.14 (data in Fig. 3B). An approximate significance level can be obtained by a comparison to the chi-square distribution with eleven degrees of freedom. This procedure yields an approximate attained significance level of $P=0.14$, so there is not sufficient evidence to reject the null hypothesis of within-replicate homogeneity of the sex ratio.

A more precise experiment could be designed for detecting genetic recognition and an associated sex-ratio shift. In the experiment described above, the number of foundresses in the maturing figs from which the wasps for introductions were reared was unknown. Wasps reared from figs in which the foundress numbers were experimentally controlled could be used, so that the expected number of sibs introduced into an experimental fig could be contolled. The proportion of sibs among the foundresses could be manipulated, and the total number of foundresses could be varied, allowing comparisons over a broad range of genetic variances within figs. Such an experiment should indicate whether the proximate cue used by the foundresses is genotypic recognition or foundress number.

\section{Extending Sex-Ratio Theory for Fig Wasps}

It is clear that the qualitative hypothesis of increasing sex ratio with increasing foundress number is supported by the data, but the previously used quantita- 
tive prediction in Equation (2) is consistently higher than the observed sex ratio (Fig. 1). There are many unrealistic assumptions underlying Equation (2), and it seems reasonable to ask, in an a posteriori fashion, how the predicted sex ratio changes when more realistic assumptions are substituted. There are many factors that might influence the sex ratio. One must decide which factors to include in the model, whether each should be treated as deterministic or stochastic, whether to treat the predicted sex ratio as conditional on different levels of each factor or responsive only to the average over all levels, and to what extent the influence of each factor added to a revised model is testable. The following discussion focuses on eleven factors that have either been mentioned by previous authors or that follow from the natural history of fig wasps. Where appropriate for fig wasps, a revised prediction is derived.

i) Sex ratio versus sex investment ratio. - The models in this paper are all concerned with numbers of the two sexes when the investment in each individual of either sex is equal, but previous work suggests that these results are also valid for the more general case of investment ratio in the two sexes (Uyenoyama and Bengtsson, 1981, 1982). Almost all the energy to produce a fig wasp comes from the plant tissues in the floret ovary. It is possible that the mother invests some limiting resource asymmetrically in the two sexes, but no data are available, nor is there any clear a priori reason to suspect a skew towards a particular sex.

ii) Variable foundress number per fig.Since the foundresses lay their eggs simultaneously within a sealed cavity $2-5$ $\mathrm{mm}$ in diameter and die inside the cavity after ovipositing, it seems likely that each foundress can assess the number of other foundresses ovipositing in that fig. The question is whether hymenopteran mothers adjust the sex ratio of their broods according to cues associated with the level of LMC, since they have the ability to adjust the sex ratio of their broods by varying the percentage of eggs that are fertilized. Three papers have incorrectly used (2) as a conditional model for varying deme sizes: Hamilton (1979) and Frank (1983b) for fig wasps, and Werren (1983) for Nasonia vitripennis. The derivation of an appropriate model for sex ratio conditional on deme size is given in Appendix I. The result is obtained by a three-level expansion of Price's $(1970,1972)$ hierarchical analysis of selection. The general approach is described in a related paper (Frank, 1985b). The predicted sex ratio, $r_{n}{ }^{*}$, in a deme of size $n$, is

$$
r_{n}^{*}=(1 / 2) R P_{d(n) t}
$$

where the prediction is an evolutionarily stable strategy, or an ESS, in the sense of Maynard Smith and Price (1973). The asymmetry of inheritance between the sexes is $R=(1+F) /(1+2 F)$ for maternal control of the sex ratio in haplodiploid organisms, and $P_{d(n) t}$ is the expected genetic variance within demes of size $n$, divided by the population variance. To derive a specific prediction, values of $F$ and $P_{d(n) t}$ are needed. When settling of foundresses into demes is random with respect to genotype in each generation, $F=h /(4-3 h)$ where $h$ is the proportion of sib mating $(\mathrm{Li}, 1976)$,

$$
h=\frac{\sum \lambda_{n}\left(1-r_{n}\right)}{\sum \lambda_{n}\left(1-r_{n}\right) n}
$$

when $\lambda_{n}$ is the frequency of demes of size $n, r_{n}$ is the sex ratio of each foundress in a deme of size $n$, each foundress produces the same brood size, and mating is random within each deme. The numerator in (4) is proportional to the number of sib-mated females produced in the population, since the number of females produced from a deme of size $n$ is proportional to $\left(1-r_{n}\right) n$ and sib mating occurs with probability $1 / n$. The denominator is proportional to the number of females produced from all demes. Thus $h$ is the proportion of sib-mated females in each generation. Notice that the amount of sib mating is proportional to the inverse of 
TABLE 1. The pooled distribution of the number of pollinating wasps per fig for six samples (size range 76-146) from Ficus citrifolia. Each sample was taken from several neighboring trees in Everglades Park, Florida, during July 1981. $n$ is the number of foundresses per fig, and $\lambda_{n}$ is the frequency of figs with $n$ foundresses among the total sample of 706 figs, excluding the nine zeros. The small number of zeros relative to a Poisson expectation can probably be explained by the long period that empty figs remain receptive to entering wasps (about $6-10$ days) when compared to the short period that figs remain receptive after the first wasp has entered (about one day). Further details in Frank (1983a).

\begin{tabular}{llccccccc}
\hline \hline$n$ & 0 & 1 & 2 & 3 & 4 & 5 & 6 & 7 \\
obs. & 9 & 375 & 188 & 87 & 35 & 17 & 3 & 1 \\
$\lambda_{n}$ & & 0.53 & 0.27 & 0.12 & 0.05 & 0.02 & 0 & 0 \\
\hline
\end{tabular}

the weighted harmonic mean of foundress numbers, agreeing with Wade $(1982 a)$. The weights for each deme size are $\lambda_{n}\left(1-r_{n}\right) n$, under the assumption that all foundresses in the population produce the same brood size, independent of the deme size $n$.

The expected variance within a deme, when settling of foundresses into figs is random, is the expected value of the sampling variance, $s^{2}$, which is $[(n-1) / n] V_{t}$ (Lindgren, 1976), where $V_{t}$ is the population variance. Thus $P_{d(n) t}=(n-1) / n$, and

$$
r_{n}^{*}=(1 / 2)[(4-2 h) /(4-h)][(n-1) / n] \text {. }
$$

Solving for $r_{n}^{*}$ yields

$$
\begin{aligned}
r_{n}^{*} & =\frac{1}{2}\left(\frac{x-\sqrt{x^{2}-4 y z}}{y}\right)\left(\frac{n-1}{n}\right) \\
x & =\sum \lambda_{n}\left(6 n^{2}-4 n+1\right) / 4 n \\
y & =\sum \lambda_{n}(4 n-1)(n-1) / 4 n \\
z & =\sum \lambda_{n}(2 n-1) n / 4 n .
\end{aligned}
$$

Notice that the form of $r_{n}{ }^{*}$ is consistent with the $(1 / 2) R P_{d t}$ of (1).

To obtain a prediction for the particular fig wasp population that I studied, values are needed for $\lambda_{n}$. I sampled figs from an area within a $10 \mathrm{~km}$ radius of the study area on six occasions during July and August 1981 (Table 1). Using these data in (6), the predicted sex ratios are plotted in Figure 1. Although the predictions from (6) are closer to the observations than the predictions from (2), the predicted sex ratios are still consistently greater than the observed sex ratios.

iii) Variable brood sizes among foundresses within a fig. - Several authors have noted that the assumption that all foundresses produce exactly the same brood size is unrealistic (Hamilton, 1979; Werren, 1980; Charnov, 1982). When brood sizes vary, "the effective deme size" is smaller than $n$, i.e., the level of inbreeding and LMC is greater than for $n$ equalsized broods. Charnov (1982) gave the predicted sex ratio for variable brood size in a diploid model, with the sex ratio unconditionally adjusted according to the effective deme size-that is, all females in a deme produce the same sex ratio (unconditional), rather than each female adjusting her sex ratio according to her relative brood size within the deme (conditional). Here I derive an analogous prediction for haplodiploid organisms when the sex ratio depends unconditionally on the variation in the brood sizes among foundresses within each fig, but is also conditionally adjusted according to deme size.

Let $K_{n}$ be the expected number of eggs per female in a deme of size $n, k_{n i}$ be the number of offspring by the $i$ th female $(i=1,2,3, \ldots, n)$ in a deme of size $n$, and $\beta_{n i}=k_{n i} / K_{n}$. Then the expected proportion of sib-mated daughters produced from a deme of size $n$ is

$$
h_{n}=\sum \beta_{n i}^{2} / n^{2}=1 / n^{\prime}=\tau_{n} / n
$$

where $n^{\prime}$ is an effective deme size $(1 \leq$ $\left.n^{\prime} \leq n\right)$, and $\tau_{n}-1$ is a measure of the 
TABLE 2. Summary of data in Figure 1, with fitted sex ratios from Equation (13). $\vec{r}_{n}$ is the average sex ratio in figs with $n$ foundresses, $K_{n}$ is the average brood size per foundress in figs with $n$ foundresses, and "fitted" $r_{n}$ is the fitted sex ratio from (13).

\begin{tabular}{lllcccc}
\hline \hline Foundresses & 1 & 2 & 3 & 4 & 5 & 8 \\
No. Observations & 9 & 9 & 11 & 11 & 12 & 5 \\
$\boldsymbol{F}_{n}$ & 0.102 & 0.125 & 0.149 & 0.182 & 0.231 & 0.276 \\
SE of mean & 0.023 & 0.016 & 0.012 & 0.009 & 0.017 & 0.036 \\
$K_{n}$ & 72.0 & 78.3 & 64.0 & 58.0 & 48.1 & 24.4 \\
SE of mean & 10.9 & 8.4 & 7.4 & 6.1 & 5.2 & 3.4 \\
Fitted $r_{n}$ from (13) & & 0.121 & 0.161 & 0.181 & 0.193 & 0.211 \\
\hline
\end{tabular}

expected variance in offspring number per female in a deme of size $n$ when standardized by $K_{n}^{2}$, the square of the average brood size per foundress in a deme of size $n$. By details given in Appendix II, $r_{n}{ }^{*}$ is the same as in (6) when $n$ is replaced by $n^{\prime}$ in the variance term, and for the calculation of $R: x, y$, and $z$ are replaced by:

$$
\begin{gathered}
x^{\prime}=\sum \lambda_{n} K_{n}\left[6\left(n^{\prime}\right)^{2}-2 n^{\prime}\left(\tau_{n}+1\right)\right. \\
\left.\quad+\tau_{n}\right] / 4 n^{\prime} \\
y^{\prime}=\sum \lambda_{n} K_{n}\left(n^{\prime}-1\right)\left(4 n^{\prime}-\tau_{n}\right) / 4 n^{\prime} \\
z^{\prime}=\sum \lambda_{n} K_{n}\left(2 n^{\prime}-\tau_{n}\right) n^{\prime} / 4 n^{\prime} .
\end{gathered}
$$

Data for $K_{n}$ are presented in Table 2 . No data are available for $\tau_{n}$. To obtain very rough estimates for $\tau_{n}$, the data for single foundress figs in Table 2 can be used. The estimated variance in brood sizes based on the nine single foundress figs is $1,069.3$. Dividing by $K_{1}{ }^{2}$ gives a standardized measure of the variance of 0.206 . Estimates of $\tau_{n}$ can be obtained from the equation $\hat{\tau}_{n}=1+[(n-1) / n]$. $(0.206)$, where $(n-1) / n$ is a small sample correction factor. The predicted sex ratios from (6) and (8), using the observed value of $K_{n}$ and the estimates for $\tau_{n}$ in $x^{\prime}, y^{\prime}$, and $z^{\prime}$ to obtain $R$, are plotted in Figure 1 . These predictions are considerably closer to the observations than (6), and the underlying assumptions seem quite plausible.

iv) Variable sex ratios among foundresses within a fig. - Werren (1980) presented a model that predicts the sex ratio of a foundress when conditional on both her relative contribution to the total brood of the deme, and on combined sex ratio of all other foundresses in the deme. Werren was studying Nasonia vitripennis, a parasitic wasp in which the first foundress lays her eggs, and then flies off. The second foundress is able to detect whether a host has already been parasitized (Wylie, 1965; Holmes, 1972) and adjusts her sex ratio according to whether she is a first or second foundress (Holmes, 1972; Werren, 1980). If a female lays eggs on a host as a second foundress, she usually lays a much smaller brood than the first foundress, and since the foundresses lay eggs sequentially, the second foundress may also respond to the usual sex ratio of single-foundress hosts, which tends to be extremely female-biased (Werren, 1980). In contrast, fig wasps lay their broods simultaneously, except for rare occasions in which a foundress dies before the last foundress has entered. I have therefore constructed a model in which foundresses can adjust their sex ratios according to the relative sizes of their broods but are not able to assess the sex ratios of the other broods.

The model assumes that a) $n$ mated females colonize each deme, and settling is random with respect to genotype, b) the number of demes in the population is infinite, c) mating and mate competition are random within each deme, and d) each female can assess her relative contribution to the total brood of the deme and adjust her sex ratio accordingly.

Let $\beta_{i}$ be the relative brood size of the $i$ th female in a deme, $i=1,2, \ldots, n$; 
$\beta_{i}=k_{n i} / K_{n}$, where $k_{n i}$ is the brood size of the $i$ th female, and $K_{n}$ is the average brood size in a deme of size $n, \Sigma \beta_{i}=n$. Let the sex ratio of the $i$ th female be $r_{i}$, and the sex ratio in the deme $r=\Sigma \beta_{i} r_{i} /$ $n$. Then, as shown in Appendix III,

$$
\beta_{i} r_{i}^{*}=(1 / 2) R[(n-1) / n]
$$

and since $\beta_{i} r_{i}=r$ for all $i$ at equilibrium, the deme sex ratio $r$ is the right hand side of (9) for all values of $\beta_{i}$ such that $r^{*}<$ $\beta_{i}<n-(n-1) r^{*}$, and $\Sigma \beta_{i}=n$. The important message is that, ignoring the asymmetric relatedness term $R$, the deme sex ratio is independent of the variance in brood sizes among the foundresses when each foundress adjusts her sex ratio only conditional on her relative contribution to the deme. To make a comparison with the fig wasp data, let $R=2 / 3$, which is its lower bound (i.e., $F=1$ ). The predicted sex ratio per deme (fig) is now given by

$$
r_{n}=(1 / 3[(n-1) / n]
$$

and as shown in Figure 1, (10) is closer to the observed sex ratios than is (6); but it is almost identical to the predictions of (8) and is still inflated relative to the data.

Werren (1980) gave a prediction for a foundress' sex ratio, $r_{2}$, conditional on both relative brood size, $\beta_{1}$, and sex ratio, $r_{1}$, of all broods other than her own. Using the notation above, he showed that

$$
r_{2}^{*}=\left(\sqrt{\beta_{1} r_{1}}-\beta_{1} r_{1}\right) /\left(2-\beta_{1}\right)
$$

where $\beta_{1}=2 k_{1} /\left(k_{1}+k_{2}\right), k_{1}$ is the total brood size of all other foundresses and $k_{2}$ is the brood size of the female of interest. This model is probably not applicable to the fig wasp data I collected, since all foundresses were introduced within a short time period and, thus, laid their eggs simultaneously.

v) Nonrandom settling of foundresses into figs. - The predicted sex ratios that have been compared to the data so far have all been based on the assumption that the foundresses settle randomly in each generation. Random settling with respect to genotype gives $P_{d(n) \ell}=(n-1) / n$, for all $n$, as in Equations (2), (6), and (9). If settling is not random, then there are two plausible mechanisms underlying the settling pattern. First, since foundresses enter a fig sequentially, prospective foundresses of a given fig might assess and react to cues associated with genotype left at the entrance to the fig by the earlier foundresses. Second, settling might be random with respect to the local subpopulation of potential foundresses, but subpopulations separated in time and space might be genetically different.

No significant preference of foundresses was detected for occupied (already entered by a previous foundress) versus unoccupied figs, figs punctured by parasite ovipositors versus those not punctured, or figs occupied by sibs versus figs occupied by non-sibs. (Details of these three experiments are presented in Frank, 1983a.) Such preferences cannot be ruled out on the basis of these crude experiments, but in the absence of any contrary evidence, I will assume that settling from the local subpopulation is genetically random.

No data are available on genetic differentiation among subpopulations of fig wasps. The common belief about such differentiation is that it is very likely to be negligible except under extreme conditions (reviewed by Wade, 1978), and such conditions are not likely for fig wasps. However, this view is derived mainly from theoretical studies, and two related points suggest that subpopulation structure should not be dismissed a priori. First, from a series of empirical studies Wade $(1982 b)$ has inferred the existence of much more selectable genetic variability among subpopulations of Tribolium than predicted by previous theory, and based on Wade's experiments, Slatkin (1981) has developed a theory of "populational heritability," which suggests that conditions for differentiation are not nearly as extreme as those required by earlier models. Second, the theoretical studies on genetic divergence among subpopulations usually ignore that selection may increase the rate of diver- 
gence. If spatial and temporal isolation of subpopulations occurs, then genes lowering the sex ratios of partially isolated groups will be favored, since lower sex ratios correspond to higher group productivity (Wilson and Colwell, 1981). Thus, partial isolation may act synergistically with selection of sex ratio among groups to further enhance differentiation.

We can take advantage of a very useful relationship for Wright's (1969) panmictic index for describing subpopulation structure

$$
P_{d(n) t}=P_{d(n) s} P_{s t}
$$

where $P_{d(n) t}$ is the within-deme genetic variance divided by the total population variance, $P_{d(n) s}$ is the within-deme variance divided by the variance of the subpopulation within which it is nested, and $P_{s t}$ is the subpopulation variance divided by the total population variance. As shown in a related paper (Frank, 1985b), a decrease in $P_{s t}$ means greater divergence among subpopulations, and hence a stronger effect of group selection. Equivalently, a decrease in $P_{s t}$ also means an increase in the genetic correlation within groups, $\rho$, and therefore a stronger effect of within-sex competition and inbreeding. (See Notes at end of paper.)

When settling from each local subpopulation into demes (figs) is random, $P_{d(n) s}=(n-1) / n$, and when there is no divergence among subpopulations, $P_{s t}=$ 1 , so $P_{d(n) t}=(n-1) / n$. In general, with random settling from subpopulations into demes, $P_{d(n) t}=[(n-1) / n] P_{s t}$. To make a comparison of the predicted sex ratio with the data, we also need a value for the asymmetric relatedness coefficient, $R=(1+F) /(1+2 F)$. Using $x^{\prime}, y^{\prime}$, and $z^{\prime}$ in (8), as in assumption iii above, unequal brood sizes within a fig and different average brood sizes as a function of $n$ are accounted for, and $R$ is estimated to be 0.720 , and so $F$ is estimated to be 0.636 . To estimate $P_{s t}$, we can now fit the model

$$
\begin{aligned}
r_{n} & =(1 / 2) R P_{d(n) t} \\
& =0.360[(n-1) / n] P_{s t}
\end{aligned}
$$

and

$$
\begin{aligned}
\ln \left(r_{n}\right)= & \ln \left(0.360 P_{s t}\right) \\
& +\ln (n-1)-\ln (n) .
\end{aligned}
$$

The sex ratio data for $n=1$ are ignored since the formal theoretical prediction is $r_{1}=0$, while the realistic prediction is that the single foundress makes just enough sons to insure that all her daughters are inseminated (Hamilton, 1967; Hartl and Brown, 1970). Using the rest of the sex ratio data, the estimate for $P_{s t}$ is 0.671 , and the fitted curve is shown in Figure 1 .

This estimated value of $P_{s t}$, while it gives a close fit of prediction with observation (Fig. 1), seems too low to be accepted as realistic in the absence of any supporting evidence or information about the dispersal pattern of the wasps. Wright (1978) reviews data on $P_{s t}$ values from natural populations of a variety of species, and 0.671 would be an extreme value relative to his data set. But selection at the enzyme loci quoted by Wright is probably weak, and just how selection might affect differentiation at sex-ratio loci is not at all understood, especially since demonstrating any heritable component of sex-ratio traits is notoriously difficult. One possibility for testing the effect of subpopulation differentiation $\left(P_{s t}\right)$ would be to compare the sex ratios of populations that could be ranked according to the extent to which they appear subdivided in time and space. A ranking could be based on the demography and phenology of the host fig trees.

vi) Nonrandom mating and mate competition within the figs. - Thus far, all quantitative predictions have assumed that mating and mate competition are random with respect to genotype in each fig. To gain some information about the pattern of mating and mate competition, I performed a simple experiment. The complete design and analysis are presented elsewhere (Frank, 1985a); here I briefly summarize the results. Males were allowed to choose between searching for mates within their natal fig, or within a foreign fig. A significant tendency was ob- 
served for males to choose their natal figs $(P=0.04$ by chi-square test of association; Frank, 1985a). If this searching bias of males were associated with a preference for mating with sibs, then $F$ would be greater than calculated above, and $R$ would be lower. However $R=0.720$ was used in fitting the model in Figure 1, which is already close to the lower bound of $R=2 / 3$. When $R=2 / 3$, the estimate for $P_{s t}$ is 0.805 , so a significant amount of differentiation must still be invoked to explain the discrepancy between observations and predictions. Increased inbreeding would also lower the predicted sex ratio by increasing the relative value of a daughter over a son, since an inbred daughter passes on twice as many parental genes as a son does (Frank, 1985b; put another way, when there is inbreeding, producing extra daughters makes available more mates for sons or other related males). A tendency for sib mating would increase the amount of competition among brothers, which would additionally lower the predicted sex ratio. However, a tendency for brothers to compete mostly among themselves does not seem very likely, since such a tendency implies that males actively avoid available mates only because they are not sisters.

If the observed tendency of males to search for mates in their natal figs were associated with a tendency to aggregate in brother bands (there is currently no evidence for this), then the implications for the predicted sex ratios would not be clear. On one side, the proportion of competitive interactions among brothers would increase: to a parent, increased competition among sons reduces the reproductive value of each son and, thus, reduces the predicted proportion of males. On the other side, one can easily imagine possible benefits to individual males that move in brother mating groups. For example, in a cooperative brother group little time may be wasted on jostling with nearby males, so an individual's reproductive success might be increased.

vii) Genetic recognition among found- resses within a fig.-For the model in which sex ratio is conditional on foundress number, $n$, the deme size is used as a proximate cue that is correlated with the relative within-deme genetic variance, $P_{d(n) r}$. If females can assess cues associated with the genotypes of their cofoundresses, then they are predicted to adjust their sex ratio conditional on the assessed level of the within-deme variance. If foundresses respond to genetic cues, then the predicted sex ratio is $r_{a}{ }^{*}=$ $(1 / 2) R P_{d(a) t}$, where $P_{d(a) t}$ is the assessed value of $P_{d t}$. An unsuccessful effort was described earlier that attempted to assay for such conditional behavior, but it should be possible to devise a more telling experiment.

Miscellaneous. - Four other factors are mentioned here that may be important for fig wasps or other parasitic Hymenoptera.

viii) Control over the sex ratio. - Maternal control of the sex ratio has been assumed throughout. If an extranuclear genetic element or parasite were able to influence the sex ratio of the wasps, the predictions would change (Hamilton, 1979). For example, a matrilineally inherited gene would favor a very femalebiased sex ratio. The host fig tree also favors a very female-biased sex ratio, since both males and females destroy a potential seed while only females carry pollen. The steady rise in the sex ratio with foundress number and low variance within a given foundress number (Fig. 1) are evidence for at least partial maternal control, but the data do not rule out sexratio bias due to the host tree or uniparentally inherited infections.

ix) Male investment in offspring; limit on the number of fertilizations a male is able to accomplish. - It has been assumed that males do not invest any limited resource in offspring. If any resource that males pass on to offspring is limited-including sperm, which would limit the number of fertilizations a male could accomplish - then the predicted sex ratios would be more male biased than above, further increasing the discrepancy 
between predictions and observations. There is no evidence that any such male resource is limited (reviewed in Frank, 1983a).

x) Local resource competition. - This is Clark's (1978) phrase for describing competition among related females for a limiting resource. The prediction is analogous to that from local mate competition: the more competition there is among female relatives, the more male-biased will be the predicted sex ratio. Each female develops in her own separate floret ovary and emerges from this ovary just before collecting pollen and leaving the fig. Since pollen does not seem to be limiting (pers. obs.), and since the tiny wasps fly off singly after emerging from their natal fig, so that sisters are unlikely to compete later for receptive figs, it seems unlikely that there is any local resource competition among sisters. The same reasoning applies to within-sex competition among males for resources other than mates. (See Notes at end of paper.)

xi) Between-sex competition among relatives. - Pickering (1980) has shown that between-sex competition for resources may bias the sex ratio in $\mathrm{Hy}$ menoptera because of the asymmetry in relatedness among male and female sibs. Comments under heading $x$ above suggest that this is not applicable to fig wasps.

\section{ACKNOWLEDGMENTS}

H. J. Brockmann, J. L. Bronstein, M. G. Bulmer, R. K. Colwell, B. Crespi, W. D. Hamilton, P. O. Lawrence, J. E. Lloyd, J. R. Lucas, P. E. Smouse, and M. K. Uyenoyama discussed these ideas with me and made helpful comments on earlier drafts of the manuscript. Financial support for my research included grants from the Alexander Bache Fund, the Theodore Roosevelt Fund, and Sigma Xi. The Department of Zoology at the University of Florida provided supplies and a research assistantship. The Everglades National Park gave permission to work within their grounds, and the Agricultural Research and Education Center of the University of Florida, in Homestead, provided housing and facilities. This project was also supported by the NIH National Research Service Awards 1-T32-GM-07544-07 and R01-GM32589 from the National Institute of General Medical Sciences.

\section{Literature Cited}

Blaustein, A. R. 1983. Kin recognition mechanisms: Phenotypic matching or recognition alleles? Amer. Natur. 121:749-754.

Bulmer, M. G., AND P. D. TAYLOR. 1980. Dispersal and the sex ratio. Nature 284:448-449.

Charlesworth, D., AND B. Charlesworth. 1978. Population genetics of partial male-sterility and the evolution of monoecy and dioecy. Heredity 41:137-153.

ـ 1981. Allocation of resources to male and female functions in hermaphrodites. Biol. J. Linn. Soc. 15:57-74.

Charnov, E. L. 1982. The Theory of Sex Allocation. Princeton Univ. Press, Princeton, NJ.

Clark, A. B. 1978. Sex ratio and local resource competition in a prosiminian primate. Science 201:163-165.

Colwell, R. K. 1981. Group selection is implicated in the evolution of female-biased sex ratios. Nature 290:401-404.

Fisher, R. A. 1941. Average excess and average effect of a gene substitution. Ann. Eugen. 11:5363.

Frank, S. A. 1983a. Theoretical and Empirical Studies of Sex Ratios, Mainly in Fig Wasps. M.S. Thesis, Univ. Florida, Gainesville.

- 1983b. A hierarchical view of sex-ratio patterns. Fla. Entomol. 66:42-75.

. 1984. The behavior and morphology of the fig wasps Pegoscapus assuetus and P. jimenezi: Descriptions and suggested behavioral characters for phylogenetic studies. Psyche 91: 289-308.

-1985a. Are mating and mate competition by the fig wasp Pegoscapus assuetus (Agaonidae) random within a fig? Biotropica 17:170-172.

—. $1985 \mathrm{~b}$. Hierarchical selection theory and sex ratios. I. General solutions for structured populations. Theoret. Popul. Biol. In press.

GaLIL, J. 1977. Fig biology. Endeavour, New Series $1: 52-56$.

Hamilton, W. D. 1967. Extraordinary sex ratios. Science 156:477-488.

- 1972. Altruism and related phenomena, mainly in social insects. Ann. Rev. Ecol. Syst. 3:193-232.

- 1979. Wingless and fighting males in fig wasps and other insects, pp. 167-220. In M. S. Blum and N. A. Blum (eds.), Sexual Selection and Reproductive Competition in Insects. Academic Press, N.Y.

HartL, D. L., ANDS. W. BRown. 1970. The origin of male haploid genetic systems and their pre- 
dicted sex ratio. Theoret. Popul. Biol. 1:165190.

Hill, D. 1967. Figs (Ficus spp.) and fig-wasps (Chalcidoidea). J. Natur. Hist. 1:413-434.

Hollander, M., AND D. A. Wolfe. 1973. Nonparametric Statistical Methods. Wiley, N.Y.

HOLMES, H. B. 1972. Genetic evidence for fewer progeny and a higher percent males when $\mathrm{Na}$ sonia vitripennis oviposits in previously parasitized hosts. Entomophaga 17:79-88.

Holmes, W. G., AND P. W. Sherman. 1982. The ontogeny of kin recognition in two species of ground squirrels. Amer. Zool. 22:491-517.

JANZEN, D. H. 1979. How to be a fig. Ann. Rev. Ecol. Syst. 10:13-51.

Lacy, R. C., and P. W. Sherman. 1983. Kin recognition by phenotypic matching. Amer. Natur. 121:489-512.

LI, C. C. 1976. A First Course in Population Genetics. Boxwood, Pacific Grove, CA.

LINDGREN, B. W. 1976. Statistical Theory. Macmillan, N.Y.

Maynard Smith, J., and G. R. Price. 1973. The logic of animal conflict. Nature 246:15-18.

NUNNEY, L. 1985. Female-biased sex ratios: Individual or group selection? Evolution 39:349361.

PICKerING, J. 1980. Larval competition and brood sex ratios in the gregarious parasitoid Pachysomoides stupidus. Nature 283:291-292.

PRICE, G. R. 1970. Selection and covariance. Nature 227:520-521.

- 1972. Extension of covariance selection mathematics. Ann. Hum. Genet. 35:485-490.

SLATKIn, M. 1981. Populational heritability. Evolution 35:859-871.

TAYLOR, P. D. 1981. Intra-sex and inter-sex sib interactions as sex ratio determinants. Nature 291:64-66.

TAYlor, P. D., AND M. G. Bulmer. 1980. Local mate competition and the sex ratio. J. Theoret. Biol. 86:409-419.

Trivers, R. L., AND H. Hare. 1976. Haplodiploidy and the evolution of social insects. Science 191:249-263.

UYeNOYAMA, M. K., AND B. O. BeNGTSSON. 1981. Towards a genetic theory for the evolution of the sex ratio II. Haplodiploid and diploid models with sibling and parental control of the brood sex ratio and brood size. Theoret. Popul. Biol. 20:57-79.

- 1982. Towards a genetic theory for the evolution of the sex ratio III. Parental and sibling control of brood investment ratio under partial sib-mating. Theoret. Popul. Biol. 22:4368.

WADE, M. J. 1978. A critical review of the models of group selection. Quart. Rev. Biol. 53:101114.

114. 1982a. The effect of multiple inseminations on the evolution of social behaviors in diploid and haplodiploid organisms. J. Thecret. Biol. 95:351-368. 1982b. Group selection: Migration and the differentiation of small populations. Evolution 36:949-961.

WERREN, J.H. 1980. Sex ratio adaptations to local mate competition in a parasitic wasp. Science 208:1157-1159.

. 1983. Sex ratio evolution under local mate competition in a parasitic wasp. Evolution 37: 116-124.

WIEBES, J. T. 1979. Co-evolution of figs and their insect pollinators. Ann. Rev. Ecol. Syst. 10:112.

12. 1983. Records and descriptions of Pegoscapus Cameron (Hymenoptera Chalcidoidea, Agaonidae). Proc. Kon. Ned. Akad. Wetensch. 86:243-253.

Wilson, D. S., ANd R. K. Colwell. 1981. Evolution of sex ratios in structured demes. Evolution 35:882-897.

WRIGHT, S. 1969. Evolution and the Genetics of Populations, Vol. 2, Univ. Chicago Press, Chicago, IL.

. 1978. Evolution and the Genetics of Populations, Vol. 4. Univ. Chicago Press, Chicago, IL.

WYLIE, H. G. 1965. Discrimination between parasitized and unparasitized house fly pupae by females of Nasonia vitripennis (Walker) (Hymenoptera: Pteromalidae). Can. Entomol. 97: 279-286.

Corresponding Editor: R. H. Crozier

\section{APPENDIX I}

\section{LMC When Deme Sizes Vary}

The assumptions and derivation of Equation (1) are given in a related paper (Frank, 1985b). Here I relax two unrealistic assumptions, so that a new model may be derived that more accurately reflects the natural history of fig wasps and similar organisms.

i) It is usually assumed that all demes were founded by exactly the same number of females, $n$ (i.e., $n$ is a constant). Instead, let demes be of size $n$ with probability $\lambda_{n}, n=1,2,3, \ldots, L$. (ii) It is usually assumed that the sex ratio is a genetically fixed trait and that each female in the population is predicted to produce the same equilibrium sex ratio, $r^{*}$. However, there is much evidence that the sex ratio in fig wasps and other Hymenoptera is a phenotypically plastic trait under maternal control (evidence reviewed by Charnov, 1982). Since the level of local mate competition within a fig varies with the number of foundresses, $n$, the best sex ratio-in terms of fitness-for a mother to produce depends on $n$. The foundresses lay their eggs simultaneously within the tiny central cavity of the fig (2-5 $\mathrm{mm}$ in diameter). So it seems plausible that each is able to assess the number of foundresses and adjust her sex ratio accordingly. Let $r_{n}$ be the sex ratio produced by a female within a deme with $n$ foundresses.

I now derive a more general result than Equation 
(1) under the relaxed conditions $i$ and ii, using Price's $(1970,1972)$ hierarchical selection theory. Application of this method for sex ratios is described fully in Frank (1985b). Assume that there are two sex-ratio phenotypes: an $a$-type that produces sex ratios $r_{a n}$, and a $b$-type that produces sex ratios $r_{b n}$, $n=1,2,3, \ldots, L$. The essence of the method is to locate values of $r_{a n}$ such that, for each $n$, no $r_{b n}$ confers a greater fitness than does $r_{a n}$. To begin the derivation, a three-level expansion of Price's equation is needed:

$$
\begin{aligned}
w \Delta q= & R\left(w_{n}, q_{n}\right) V\left(q_{n}\right) \\
& +\sum_{n} \lambda_{n}\left[R_{n}\left(w_{n d}, q_{n d}\right) V_{n}\left(q_{n d}\right)\right] \\
& +\sum_{n} \sum_{d} \lambda_{n} \alpha_{d}^{(n)}\left[R_{n d}\left(w_{n d i}, q_{n d i}\right) V_{n d}\left(q_{n d i}\right)\right]
\end{aligned}
$$

where $w$ is fitness and $q$ is the frequency of $b$-types, $n$ is the number of foundresses per deme, $n d$ is the number of $b$-types in a deme with $n$ foundresses, $n d i$ is the $i$ th individual foundress in an $n d$-type group, $i=1,2, \ldots, n, \lambda_{n}$ is the proportion of demes with $n$ foundresses, and $\alpha_{d}^{(n)}$ is the proportion of demes of a fixed size $n$ of which $d$ members are $b$-types. $q_{n d i}$ is the frequency of $b$-types within the $n d i t h$ individual, and so is unity if ndi is a $b$-type, and zero if an $a$-type. $q_{n d}=d / n$, and $q_{n}=\sum_{d} \alpha_{d}^{(n)} q_{n d}$. $w_{n d i}$ is the fitness of the ndith individual, and, by the reasoning described in Frank (1985b) for the constant deme size model, the relative fitness of an $a$-type is

$w_{n d i}(a)=r_{a n}\left[\left(1-r_{d n}\right) / r_{d n}\right] B_{p s}+\left(1-r_{a n}\right) B_{p d}$

where $r_{d n}=\left(1-q_{d n}\right) r_{a n}+q_{d n} r_{b n}=r_{a n}-\Omega_{n} q_{d n}, \Omega_{n}=$ $r_{a n}-r_{b n}$, and $B_{p s}$ and $B_{p d}$ are defined in the text. $w_{n d}=\left(1-q_{n d}\right) w_{n d i}(a)+q_{n d} w_{n d i}(b)$, where $w_{n d i}(b)$ is the same as (A2) with $b$ replacing $a . R(w, q)$ expressions are regressions of fitness on the frequency of $b$-types, and $V_{n d}\left(q_{n d i}\right)$ is the within-deme variance $\left(1-q_{n d}\right) q_{n d}, V_{n}\left(q_{n d}\right)$ is the variance among demes of fixed size $n$, and $V\left(q_{n}\right)$ is the variance among demes of different sizes. Defining $B=B_{p s}+B_{p d}$ and continuing as in the constant deme size derivation (Frank, 1985b),

$$
\begin{gathered}
w \Delta q=\sum_{n} \Omega_{n}\left(1-r_{a n}+\Omega_{n} q_{n}\right)\left(q_{n}-q\right) B \\
+\sum_{n} \Omega_{n} B V_{n}\left(q_{n d}\right) \\
-\sum_{n} \sum_{d} \lambda_{n} \alpha_{d}^{(n)}\left(B_{p s} / r_{d n}-B\right) \\
\cdot V_{n d}\left(q_{n d i}\right) .
\end{gathered}
$$

Taking the derivative of $\bar{w} \Delta q$ with respect to $r_{b n}$ and setting the derivative equal to zero at $r_{a n}=r_{b n}=r^{*}$ yields the system of equations:

where

$$
r_{n}^{*}=(1 / 2) R P_{d(n) t} \quad n=1,2, \ldots, L
$$

$$
P_{d(n) r}=\sum_{d} \alpha_{d}^{(n)} V_{n d}\left(q_{n d i}\right) / V_{t}
$$

the variance within demes of size $n$ divided by the total variance.

\section{APPENDIX II}

\section{LMC When Brood Sizes Vary within a Deme}

The details leading from Equation (7) to Equation (8) are given in this appendix. The proportion of sib matings in the population is

$$
h^{\prime}=\frac{\sum \lambda_{n} K_{n}\left(1-r_{n}\right) \tau_{n}}{\sum \lambda_{n} K_{n}\left(1-r_{n}\right) n} .
$$

To obtain the expected variance within a deme, it is convenient to use Wright's (1969) notation, where $P_{d(n) t}=1-F_{d(n) t}$, and $F_{d(n) t}$ is the additive genotypic correlation among parents of interacting individuals within a deme of size $n$. While it has not been proved that the correlation (or perhaps regression coefficient of relatedness) among parents of interacting individuals is the correct measure for $F_{d(n) t}$ for sex-ratio predictions, the results below have been derived by standard recurrence relation techniques for single locus models (Frank, 1983a), and this method has also given results that agree with more traditional methods in a variety of other scenarios (Frank, 1985b). With random settling of foundresses into demes, $F_{d(n) t}$ is the proportion of interactions among sibs, $\Sigma \beta^{2}{ }_{n i} / n^{2}=1 / n^{\prime}$. Hence, under random settling, $P_{d(n) t}=\left(n^{\prime}-1\right) / n^{\prime}$, which may be interpreted as the expected sampling variance for the effective deme size, $n^{\prime}$, divided by the population variance. $r_{n}^{*}$ is now obtained by replacing $h$ with $h^{\prime}$, and $n$ with $n^{\prime}$ in Equations (5). Solving for $r_{n}{ }^{*}$ yields the same result as (6) when $n$ is replaced by $n^{\prime}$ in the variance term, and $x, y$, and $z$ are replaced by $(8)$ in the text.

\section{APPENDIX III \\ LMC When Sex Ratios and Brood Sizes Vary within a Deme}

The derivation of (9) is given in this appendix. Let $\beta_{i}$ be the relative brood size of the $i$ th female in a deme, $i=1,2, \ldots, n ; \beta_{i}=k_{n i} / K_{n}$, where $k_{n i}$ is the brood size of the $i$ th female, and $K_{n}$ is the average brood size in a deme of size $n, \Sigma \beta_{i}=n$. Let the sex ratio of the $i$ th female be $r_{i}$, and the sex ratio in the deme $r=\Sigma \beta_{i} r_{i} / n$. The relative number of inseminations by sons of the $i$ th female is proportional to $\beta_{i} r_{i} / r$, the number of females to be inseminated is proportional to $1-r$, and the relatedness of mother to son is $B_{p s}$, so the genetic value of a son is proportional to $\beta_{i} r_{i}[(1-r) / r] B_{p s}$. The number of grandchildren through daughters is proportional to $\beta_{i}\left(1-r_{i}\right)$, and the relatedness of mother to daughter is $B_{p d}$, so relative fitness of the $i$ th female is

$$
w_{i}=\beta_{i} r_{i}[(1-r) / r] B_{p s}+\beta_{i}\left(1-r_{i}\right) B_{p d} .
$$

Since we seek the value of $r_{i}$ that maximizes $w_{i}$, 
$\frac{\partial w_{i}}{\partial r_{i}}=\left[\beta_{i} r_{i}\left(-\beta_{f} / n r^{2}\right)+\beta_{i}(1 / r-1)\right] B_{p s}-\beta_{i} B_{p d}=0$

yielding

$$
\beta_{i} r_{i} R=n r R-2 n r^{2},
$$

since $R=2 B_{p s} / B$, where $B=B_{p s}+B_{p d}$.

Rearranging, with $r=\sum \beta_{i} r_{i} / n$,

$$
\sum \beta_{i} r_{i}=\sqrt{R n \sum_{j+i} \beta_{j} r_{j} / 2}
$$

For $n=2$,

$$
\beta_{1} r_{1}+\beta_{2} r_{2}=\sqrt{\beta_{1} r_{1} R}=\sqrt{\beta_{2} r_{2} R},
$$

and similarly it can be shown for any $n$ that $\beta_{i} r_{i}=$ $\beta_{j} r_{j}$ for all $i, j=1,2, \ldots, n$. Hence, $r=\sum \beta_{i} r_{i} / n=$ $\beta_{i} r_{i}$ for all $i$. Substituting into (A4) and solving yields Equation (9), subject to the restriction that $r^{*}<$ $\beta_{i}<n-(n-1) r^{*}$.

Notes Added in Proof.-i) E. A. Herre (1985 [Science 228:896-898]) has recently published data on fig wasp sex ratios for three Panamanian species and has presented a theoretical analysis related to the one described here. ii) M. G. Bulmer (1985 [Heredity, In press]) has shown that when there is a fixed number of breeding sites and many more mated females than sites, the predicted sex ratio is essentially independent of $P_{s t}$. This comes about since an increasing level of LMC and inbreeding, which favors more females, is counterbalanced by an increasing level of competition among related females for breeding sites (i.e., local resource competition), which favors fewer females. My model assumes that breeding sites are not limiting, and thus there is no local resource competition. In nature, fig wasps fall between these two extremes (see Tables 1 and 2). Therefore, the rate of change in the predicted sex ratio as a function of $P_{s t}$ may be less than the linear rate predicted in the section on nonrandom settling of foundresses. 